\title{
¿Y AHORA QUÉ? FUTURO DE LA EVALUACIÓN FORMATIVA EN EL ÁMBITO UNIVERSITARIO
}

What now? The future of formative assessment in Higher Education

E agora? O futuro da avaliação formativa no Ensino Superior

Ángel Pérez Pueyo (1)

Carlos Gutiérrez García (2)

David Hortigüela Alcalá (3)

(1) Universidad de León. Teléfono:+34 619867767. Correo electrónico: angel.perez.pueyo@unileon.es

(2) Universidad de León. Teléfono: +3498729 Correo electrónico: cgutg@unileon.es

(3) Universidad de Burgos. Teléfono:+34 947259517. Correo electrónico: dhortiguela@ubu.es

\section{Resumen}

En este trabajo defendemos la necesidad de introducir la secuenciación de competencias en el trabajo con metodologías activas y procesos de evaluación formativa. Esta secuenciación debe permitir que los estudiantes mejoren su nivel de competencia de forma sólida, gradual y coordinada entre las diversas asignaturas que componen los planes de estudios universitarios. A modo de ejemplo, presentamos el trabajo que se está desarrollando en el Grado en Ciencias de la Actividad Física y del Deporte de la Universidad de León.

Palabras clave: Competencias; secuenciación; evaluación formativa; metodologías activas

\begin{abstract}
In this work we defend the need to introduce the sequencing of competencies when working with active methodologies and formative assessment processes. This sequencing should allow students to improve their level of competence in a solid, gradual and coordinated way among the various subjects that make up the university curriculum. As an example, we outline the work that is being developed in the Degree in Physical Activity and Sports Sciences of the University of León.

Keywords: Competences; sequencing; formative assessment; active methodologies
\end{abstract}




\section{Resumo}

Neste trabalho defendemos a necessidade de introduzir o sequenciamento de competências no trabalho com metodologias ativas e processos de avaliação formativa. Esse sequenciamento deve permitir que os alunos melhorem seu nível de competência de maneira sólida, gradual e coordenada entre as várias matérias que compõem o currículo universitário. Como exemplo, apresentamos o trabalho que está sendo desenvolvido no Grau em Ciências da Atividade Física e Esportes da Universidade de León.

Palavras-chave: Competências; sequenciamento; avaliação formativa; metodologias ativas

\section{Introducción}

Durante las últimas décadas, la evaluación formativa ha demostrado no sólo su importancia en el proceso de aprendizaje sino la mejora en los resultados obtenidos por el alumnado (Biggs, 2005; Boud \& Falchikov, 2007; López-Pastor \& Pérez-Pueyo, 2017). Cuando un docente ha conseguido implantar ésta en su quehacer cotidiano para implicar al alumnado en su propio proceso de aprendizaje, el siguiente paso (aunque algunas veces se produce de manera simultánea) es la incorporación de un cambio metodológico enfocado hacia las metodologías activas. En este sentido, el aprendizaje cooperativo con sus innumerables técnicas y estrategias, el aprendizaje basado en proyectos, el aprendizaje basado en problemas o el flipped classroom son sólo algunas de las propuestas que más auge están teniendo en los últimos años, produciendo toda esta innovación una mejora evidente en los objetivos de aprendizaje planteados (Barraza, 2005; Sein-Echaluce, Fidalgo-Blanco \& Alves, 2016).

Y cuando ambos pasos se hayan dado, la implementación de un proceso de evaluación formativa y la estructuración de las clases con metodologías activas,

¿Qué nos queda por hacer?

¿Por qué en numerosas ocasiones, después de tanto esfuerzo por parte del docente, no se observa un cambio proporcional en cuanto al nivel de competencia del alumnado universitario?

¿Y ahora qué? ¿Cuál es el futuro de la evaluación formativa en el ámbito universitario? 


\section{Contextualización}

La experiencia se viene llevando a cabo en las asignaturas de Fundamentos de la Didáctica de la Actividad Física en el tercer curso y en Diseño y Análisis de la Enseñanza de La Actividad Física, ambas del Grado de Ciencias de la Actividad Física y del Deporte de la Universidad de León; y posteriormente coordinada con la asignatura de Aprendizaje de las Materias del Módulo de Educación Física del Máster Universitario de Formación de Profesorado en Educación Secundaria Obligatoria y Bachillerato, Formación Profesional y Enseñanzas de Idiomas. Las tres asignaturas son cuatrimestrales.

\section{Justificación}

Todos aquellos docentes que hayan llevado a cabo procesos de evaluación formativa asociados a la aplicación de autoevaluaciones y coevaluación, que hayan implementado metodologías activas o simplemente los que hayan desarrollado planteamientos de realización de trabajos escritos con búsquedas bibliográficas y exposiciones de los mismos (López-Pastor \& Pérez-Pueyo, 2017) habrán comprobado como estas habilidades y competencias que debe desarrollar el alumnado se repiten curso tras curso. Todas ellas son competencias denominadas transversales, como: a) la Expresión escrita, b) asociada estrechamente a la Habilidad para la búsqueda de información, y en mucho con c) el Trabajo en equipo, relacionado directamente con d) las Habilidades de relación interpersonal, y posteriormente con e) la Expresión oral; entre otras, como contempla la Universidad de León (FCAFD, 2011). Son estas competencias en las que se centra nuestro trabajo.

Pero el problema que padecemos en la universidad es el mismo que en el marco no universitario; y no es otro que la falta de concreción del nivel de competencia que deben alcanzar los alumnos en cada momento de su proceso de aprendizaje (Pérez-Pueyo, 2018; Pérez-Pueyo, Casado \& Hortigüela, 2019). Por ello, al igual que en el ámbito no universitario se han planteado en determinados entornos propuestas de secuenciación de las competencias (Pérez-Pueyo \& Casanova, 2010; Grupo Actitudes, 2015; PérezPueyo, et al., 2013) (Figura 1), en el ámbito universitario deberíamos también diseñar una propuesta de Secuenciación de las Competencias Transversales para poder establecer una contribución desde las diferentes materias de forma coordinada y sistemática (Tabla 1). 


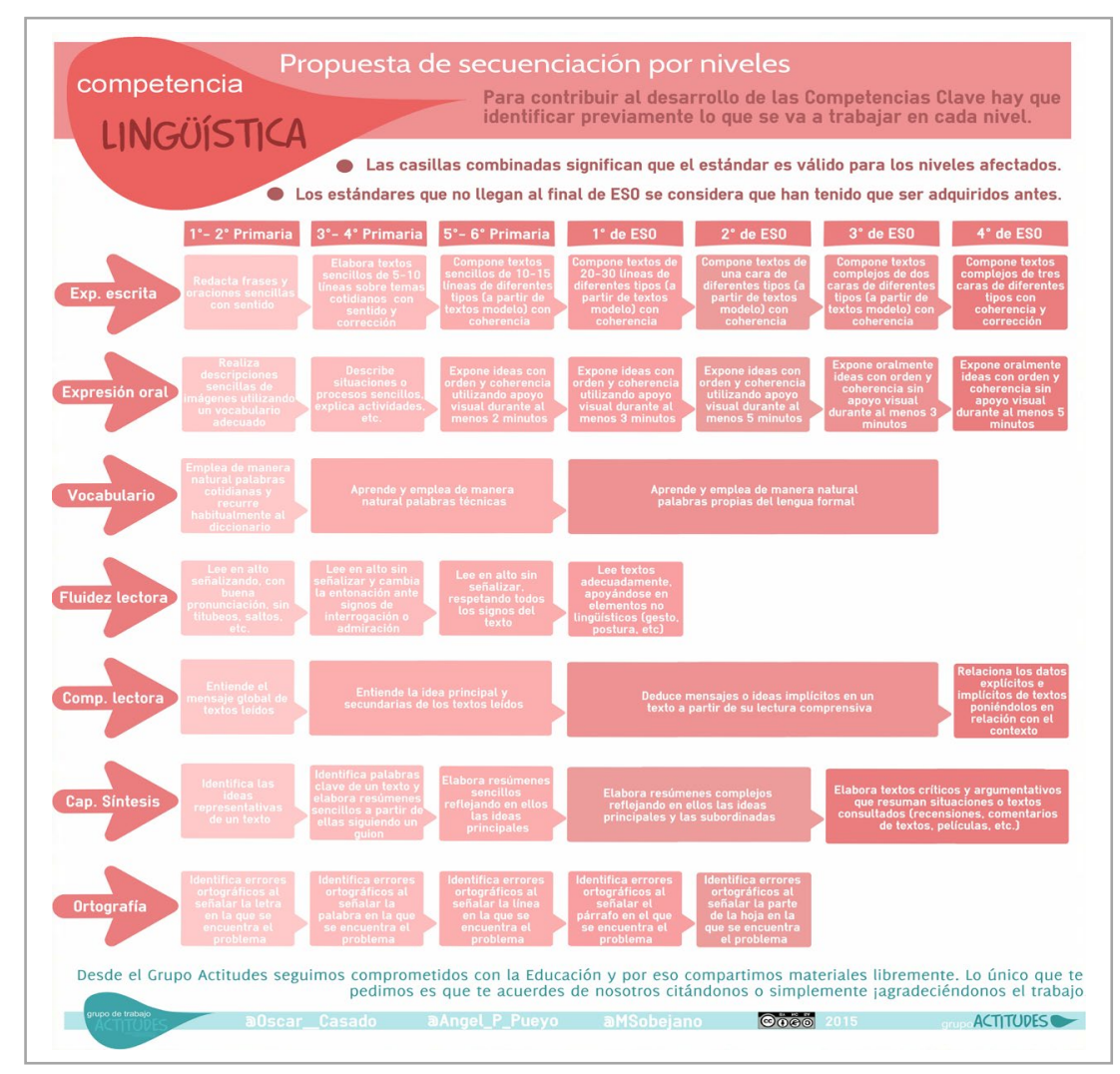

Figura 1.

Secuenciación de la competencia lingüistica del Grupo Actitudes (2015)

Tabla 1.

Ejemplo de secuenciación de la dimensión/subcompetencia expresión oral de la competencia lingüística

\begin{tabular}{|c|c|c|c|}
\hline & $1^{\circ}$ y $2^{\circ}$ de Grado & $3^{\circ}$ y $4^{\circ}$ de Grado & Máster \\
\hline 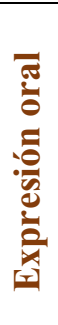 & $\begin{array}{l}\text { Expone de manera clara producciones } \\
\text { (trabajos individuales, grupales, informes, } \\
\text { búsquedas de información, ...) con orden } \\
\text { y coherencia utilizando algún soporte } \\
\text { físico para él (resumen, tarjetas, guion, ...) } \\
\text { y apoyo visual para el espectador durante, } \\
\text { al menos, 3-5 minutos. }\end{array}$ & $\begin{array}{l}\text { Expone de manera sintética y clara } \\
\text { producciones (trabajos individuales, } \\
\text { grupales, informes, búsquedas de } \\
\text { información, ...) con orden y } \\
\text { coherencia utilizando algún apoyo } \\
\text { visual sólo para el espectador durante, } \\
\text { al menos, 5-10 minutos. }\end{array}$ & $\begin{array}{l}\text { Demuestra habilidad en la } \\
\text { exposición oral de manera clara, } \\
\text { sintética y cercana al auditorio, } \\
\text { tanto en contextos de aula como } \\
\text { de patio demostrando preparación } \\
\text { para la labor docente. }\end{array}$ \\
\hline
\end{tabular}

Por ello, si verdaderamente pretendemos desarrollar estas competencias de manera clara, evidente y coherente será necesario determinar un proceso de concreción de las mismas que permita dos cuestiones fundamentales: 1) determinar el tipo y grado de aprendizaje de la competencia transversal, y 2) desarrollar actividades e instrumentos de evaluación que faciliten la contribución a las competencias desde las diferentes materias. 


\section{Diseño, desarrollo y evaluación}

La propuesta se concibe como: a) la descripción de la actividad, incluyendo su evaluación, más adecuada para trabajar y valorar la competencia (o el criterio de evaluación o resultado de aprendizaje que se pretende constatar); b) la selección de instrumentos que mejor recoja la información sobre el aprendizaje a valorar (rúbrica, escala de valoración, graduada,...); c) la organización de los momentos adecuados para proporcionar su carácter formativo; d) los procedimientos de evaluación más acordes con la actividad; y e) el proceso de evaluación compartida y de calificación dialogada, si fuese el caso.

En nuestro caso, se diseñó una secuenciación de competencias relacionadas fundamentalmente con el trabajo en equipo, la elaboración de trabajos grupales y la expresión oral. Para la evaluación utilizamos instrumentos tipo rúbricas o escalas de valoración, progresivamente más complejas, que se aplicaron en diversos momentos dentro de los proyectos que desarrollaron los estudiantes (fundamentalmente, diseño y desarrollo de sesiones y de unidades de enseñanza). Así, por ejemplo, en las exposiciones orales utilizamos una escala de valoración diferenciada según los cursos (véase una de ellas en Pérez-Pueyo, et al, 2018). Estas escalas tienen mayores niveles de concreción a nivel de máster, y todas ellas proporcionan flexibilidad en la valoración de cada uno de sus aspectos. Esta evolución de los instrumentos permite trabajar de forma sólida y progresiva en los diversos cursos.

\section{Conclusiones}

Una vez implantados a nivel docente los procesos de evaluación formativa e integración de metodologías activas en el aula, se antoja imprescindible salir de la misma y comenzar a coordinarse a nivel de curso, grado y etapa si se pretende dar coherencia y transferencia al conocimiento y al aprendizaje proporcionado.

\section{Referencias}

Biggs, J. (2005). Calidad del aprendizaje universitario. Madrid: Narcea.

Boud, D. \& Falchikov, N. (2007). Rethinking Assessment in Higher Education. Learning for the long term. Oxon: Routledge.

FCAFD (2011) Memoria para la verificación del título de Grado en Ciencias de la Actividad Física y del Deporte. Recuperado de 
https://seguimiento.calidad.unileon.es/descargas/MEMORIA-143-13-47-201101-05-10-21-24.pdf

Grupo Actitides (2015). Secuenciación de Competencias Clave. Recuperado de Ley Orgánica 2/2006, de 3 de mayo, de Educación. Boletín Oficial del Estado, 106, de 4 de mayo de 2006.

Ley Orgánica 8/2013, de 9 de diciembre, para la Mejora de la Calidad Educativa. Boletín Oficial del Estado, 295, de 10 de diciembre de 2013.

López-Pastor, V. M., \& Pérez-Pueyo, A. (2017). Aclaración de los términos implicados en el proceso de evaluación educativa. E, V. M. López-Pastor \& Á. PérezPueyo, A. (coords.), Evaluación formativa y compartida en Educación: experiencias de éxito en todas las etapas educativas (pp. 70-91). León: Universidad de León. Recuperado de http://buleria.unileon.es/handle/10612/5999

Pérez-Pueyo, A. (coord.) (2013). Qué son las Competencias Básicas y cómo se trabajan en España. Diferentes maneras de llevarlas de la teoría al aula. Hacia el Proyecto INCOBA. León: Actitudes Profesionales S. L.

https://drive.google.com/file/d/0B6zM2sfGO-LsX0I0VGdqS1VKYjQ/view

Pérez-Pueyo, A. (2018). La formación y el perfil del profesorado, reflexiones desde la teoría y la práctica. Cuadernos de Pedagogía, (489), 102-106

Pérez-Pueyo, A \& Casanova, P. (2010). La Programación de las Competencias Básicas en la Educación Secundaria Obligatoria: una propuesta de secuenciación por cursos. Madrid: CEP.

Pérez-Pueyo, A., Gutiérrez-García, C., Hortigüela, D., Hernando, Herrán, ...Costeja, G (2018). Escala de valoración para la exposición oral con Pecha-Kucha. Recuperado

de https://drive.google.com/file/d/1kK4IgMWBe8zssYMWORTmkKh7FJlCkoQr/v iew

Pérez-Pueyo, A., Gutiérrez-García, C., Hortigüela, D., Hernando, Herrán, ...Costeja, G (2019). Escala de valoración para la exposición oral con Pecha-Kucha (versión 2.0). Recuperado de https://drive.google.com/file/d/1KbZESj_W-3SFZ_jRZ6GQRNijI2fnhAM/view 